\title{
Multiscale studies of complex magnetism of nanostructures based on first principles
}

\author{
A. Antal, B. Lazarovits, L. Balogh, L. Udvardi* and L. Szunyogh \\ Department of Theoretical Physics, Budapest University of Technology and Economics, \\ Budafoki u. 8., H-1111 Budapest, Hungary
}

(Received 20 May 2008; final version received 16 June 2008)

\begin{abstract}
We present a first principles formulation that allows the derivation of parameters of a classical spin-model. In particular, relativistic effects are included, inducing tensorial exchange interactions and on-site anisotropy. Three applications of the method are shown: (i) by using a mean field treatment of the spin-model we calculate the temperature dependence of the magnetic anisotropy energy of a $\mathrm{Co}_{3} \mathrm{Cu}(001)$ film; (ii) in terms of Monte Carlo simulations for an $\mathrm{Fe}_{2} / \mathrm{W}(110)$ film we demonstrate the sensitivity of the magnetic ground state to the layer relaxations; and (iii) we also investigate the magnetic ground state of an equilateral $\mathrm{Cr}$ trimer on top of a $\mathrm{Au}(111)$ surface. These examples clearly highlight the importance of the anisotropic exchange couplings and of the Dzyaloshinsky-Moriya interactions in studying and understanding the magnetism of nanostructures.
\end{abstract}

Keywords: magnetic anisotropy; exchange interactions; magnetic nanostructures; magnetic nanoparticles; magnetic properties; thin films

\section{Introduction}

In the last decade studies of magnetic properties in confined geometries such as multilayers and clusters have led to discoveries of exciting new magnetic properties like giant magnetoresistance (GMR), exchange biasing and spin-dependent tunnelling which have attracted much attention because of their importance in magnetic storage technologies. Some of these novel properties have already found use in hard disk heads, some have the potential to do so in magnetic memories and are opening a new frontier of spintronics. The development of nanoscale devices based on electron spin requires both a fundamental understanding of magnetic interactions and practical solutions to a variety of challenges.

Multiscale approaches based on a first principles evaluation of the model parameters are very useful to study both the ground state and the dynamics of magnetic systems. There are quite a number of different methods applied in the literature to determine the exchange coupling parameters entering a model spin-Hamiltonian from first principles. Using the so-called frozen magnon approximation the spin configuration is constrained to a spin-wave of wavevector $\mathbf{q}$ and the energy of this spin-wave is calculated by employing

\footnotetext{
*Corresponding author. Email: udvardi@phy.bme.hu
} 
the generalized Bloch theorem for a spin-spiral configuration [1]. The calculated spin-wave energies are then easily mapped to a model spin-Hamiltonian [2-4]. Within the approach frequently referred to as the torque method or the method of infinitesimal rotations [5], the coupling constants $J_{i j}$ are calculated directly from the change of the energy associated with constrained rotations of the spin-polarization axes at sites $i$ and $j$. Using the magnetic force theorem $[5,6]$ the change of the total energy is approximated by the corresponding change of the one-particle energies remarkably simplifying the calculations.

The electronic and magnetic properties of thin films and deposited clusters are quite different from those of bulk. In particular, the reduction of the symmetry often results in an enhancement of the magnetic anisotropy energy (MAE) due to the spin-orbit coupling. The inclusion of spin-orbit coupling induced effects makes it necessary to introduce on-site anisotropy and tensorial couplings in the Heisenberg spin-model,

$$
\mathcal{H}=\frac{1}{2} \sum_{i \neq j} \boldsymbol{\sigma}_{i} \mathcal{J}_{i j} \boldsymbol{\sigma}_{j}+\sum_{i} K\left(\boldsymbol{\sigma}_{i}\right),
$$

where $\boldsymbol{\sigma}_{i}$ is a classical unit vector parallel to the spin-magnetic moment at site $i, \mathcal{J}_{i j}$ is a $3 \times 3$ matrix comprising the exchange interactions and $K\left(\sigma_{i}\right)$ stands for the on-site anisotropy energy. The exchange tensor, $\mathcal{J}_{i j}$, can be decomposed into three terms as [7]

$$
\mathcal{J}_{i j}=J_{i j} \mathcal{I}+\mathcal{J}_{i j}^{S}+\mathcal{J}_{i j}^{A}
$$

where $J_{i j}$ is the isotropic part of the exchange tensor,

$$
J_{i j}=\frac{1}{3} \operatorname{Tr}\left(\mathcal{J}_{i j}\right)
$$

the traceless symmetric anisotropic exchange tensor, $\mathcal{J}_{i j}^{S}$ is defined by

$$
\mathcal{J}_{i j}^{S}=\frac{1}{2}\left(\mathcal{J}_{i j}+\mathcal{J}_{i j}^{T}\right)-J_{i j} \mathcal{I},
$$

where $T$ denotes the transpose of a matrix. The antisymmetric exchange matrix, $\mathcal{J}_{i j}^{A}$, is then given by

$$
\mathcal{J}_{i j}^{A}=\frac{1}{2}\left(\mathcal{J}_{i j}-\mathcal{J}_{i j}^{T}\right)
$$

and the corresponding inter-site exchange interaction can be cast in the following form,

$$
\boldsymbol{\sigma}_{i} \mathcal{J}_{i j}^{A} \boldsymbol{\sigma}_{j}=\mathbf{D}_{i j}\left(\boldsymbol{\sigma}_{i} \times \boldsymbol{\sigma}_{j}\right),
$$

which is nothing but the well-known relativistic Dzyaloshinsky-Moriya (DM) interaction [8-10].

In this work we present some applications of the relativistic torque method [7]. First, we provide a brief summary of the method. The approach is then applied to calculate the temperature dependence of the MAE of a $\mathrm{Co}_{3} / \mathrm{Cu}(100)$ film. In case of an $\mathrm{Fe}_{2} / \mathrm{W}(110)$ film the effect of the lattice relaxation is discussed in conjunction with the DzyaloshinskyMoriya interactions. Finally, the results of the torque method and a direct mapping are compared for an equilateral $\mathrm{Cr}$ trimer deposited on $\mathrm{Au}(111)$. 


\section{Relativistic extension of the torque method}

Expressions for the derivatives of free energy with respect to the orientation of the magnetic moments can be derived within the framework of the relativistic spin-polarized Korringa-Kohn-Rostoker (RSP-SKKR) method which was successfully applied to calculate the magnetic anisotropy energies of thin films [11]. Within the magnetic force theorem, the free energy (grand potential) at zero temperature, $\mathcal{F}$, is approximated by

$$
\mathcal{F}=-\int_{-\infty}^{E_{F}} \mathrm{~d} \varepsilon N(\varepsilon),
$$

where $E_{F}$ denotes the Fermi-energy and $N(\varepsilon)$ is the integrated DOS. Employing Lloyd's formula [12], apart from a constant term corresponding to the potential-free system, the free energy can be written as

$$
\mathcal{F}=-\frac{1}{\pi} \operatorname{Im} \int_{-\infty}^{E_{F}} \mathrm{~d} \varepsilon \operatorname{Tr} \ln \tau(\varepsilon)
$$

where the site-angular momentum representation of the scattering path operator, $\tau(\varepsilon)=$ $\left\{\tau_{i j}(\varepsilon)\right\}$, can be expressed in terms of a site-diagonal single-site scattering matrix, $\mathbf{t}(\varepsilon)=\left\{t_{i}(\varepsilon) \delta_{i j}\right\}$, and the structure constants, $\mathbf{G}_{0}(\varepsilon)=\left\{G_{0, i j}(\varepsilon)\left(1-\delta_{i j}\right)\right\}$, as

$$
\tau(\varepsilon)=\left(\mathbf{t}^{-1}(\varepsilon)-\mathbf{G}_{0}(\varepsilon)\right)^{-1} .
$$

Here, $\tau_{i j}(\varepsilon), t_{i}(\varepsilon)$ and $G_{0, i j}(\varepsilon)$ are matrices in angular momentum space.

The second order derivatives of the free energy with respect to the transverse components of the magnetic moments at sites $i$ and $j, \alpha_{i} \in\left\{\vartheta_{i}, \varphi_{i}\right\}$ and $\beta_{j} \in\left\{\vartheta_{j}, \varphi_{j}\right\}$, as it is explained in [7], are

$$
\begin{aligned}
\frac{\partial^{2} \mathcal{F}}{\partial \alpha_{i} \partial \beta_{i}} & =-\frac{1}{\pi} \operatorname{Im} \int_{-\infty}^{E_{F}} \mathrm{~d} \varepsilon \operatorname{Tr}\left\{-\tau_{i i}(\varepsilon) m_{i}^{\alpha \beta}(\varepsilon)+\tau_{i i}(\varepsilon) m_{i}^{\alpha}(\varepsilon) \tau_{i i}(\varepsilon) m_{i}^{\beta}(\varepsilon)\right\}, \\
\frac{\partial^{2} \mathcal{F}}{\partial \alpha_{i} \partial \beta_{j}} & =-\frac{1}{\pi} \operatorname{Im} \int_{-\infty}^{E_{F}} \mathrm{~d} \varepsilon \operatorname{Tr}\left\{\tau_{j i}(\varepsilon) m_{i}^{\alpha}(\varepsilon) \tau_{i j}(\varepsilon) m_{j}^{\beta}(\varepsilon)\right\},
\end{aligned}
$$

where $m_{i}^{\alpha}=\frac{\partial}{\partial \alpha}\left(t_{i}^{-1}(\vartheta, \varphi)\right)$ and $m_{i}^{\alpha \beta}=\frac{\partial^{2}}{\partial \alpha \partial \beta}\left(t_{i}^{-1}(\vartheta, \varphi)\right)$. In case of spherically symmetric effective potentials and fields, the $t$-matrices corresponding to an orientation of the magnetization $(\vartheta, \varphi)$ can be expressed from those related to a quantization axis along the $z$ axis and the rotation matrices, $R(\vartheta, \varphi)$, thus the above derivatives can easily be performed.

The exchange matrix can be determined by calculating the derivatives of the free energy, Equations (10) and (11), in ferromagnetic states with three orthogonal directions of the magnetization, say, $x, y$ and $z$, and comparing the results to the derivatives of the model Hamiltonian given by Equation (1). We then get the following results for a magnetization parallel to the $x$ direction

$$
\frac{\partial^{2} \mathcal{F}}{\partial \varphi_{i} \partial \varphi_{k}}=J_{i k}^{y y}, \quad \frac{\partial^{2} \mathcal{F}}{\partial \vartheta_{i} \partial \vartheta_{k}}=J_{i k}^{z z}, \quad \frac{\partial^{2} \mathcal{F}}{\partial \varphi_{i} \partial \vartheta_{k}}=-J_{i k}^{y z}, \quad \frac{\partial^{2} \mathcal{F}}{\partial \vartheta_{i} \partial \varphi_{k}}=-J_{i k}^{z y},
$$


for a magnetization parallel to the $y$ direction

$$
\frac{\partial^{2} \mathcal{F}}{\partial \varphi_{i} \partial \varphi_{k}}=J_{i k}^{x x}, \quad \frac{\partial^{2} \mathcal{F}}{\partial \vartheta_{i} \partial \vartheta_{k}}=J_{i k}^{z z}, \quad \frac{\partial^{2} \mathcal{H}}{\partial \varphi_{i} \partial \vartheta_{k}}=J_{i k}^{x z}, \quad \frac{\partial^{2} \mathcal{F}}{\partial \vartheta_{i} \partial \varphi_{k}}=J_{i k}^{z x},
$$

and for a magnetization parallel to the $z$ direction

$$
\frac{\partial^{2} \mathcal{F}}{\partial \varphi_{i} \partial \varphi_{k}}=J_{i k}^{y y}, \quad \frac{\partial^{2} \mathcal{H}}{\partial \vartheta_{i} \partial \vartheta_{k}}=J_{i k}^{x x}, \quad \frac{\partial^{2} \mathcal{F}}{\partial \varphi_{i} \partial \vartheta_{k}}=J_{i k}^{y x}, \quad \frac{\partial^{2} \mathcal{F}}{\partial \vartheta_{i} \partial \varphi_{k}}=J_{i k}^{x y} .
$$

The second order on-site anisotropy matrices, $\mathcal{K}_{i}$,

$$
K\left(\boldsymbol{\sigma}_{i}\right)=\boldsymbol{\sigma}_{i} \mathcal{K}_{i} \boldsymbol{\sigma}_{i}
$$

can also be obtained in a similar manner. However, in particular, in case of a low pointgroup symmetry or even in the absence of a point-group symmetry of the system this procedure can be remarkably complicated. In case of uniaxial symmetry, the corresponding expressions are given in [7].

\section{Applications}

\subsection{Temperature dependent anisotropy of $\mathrm{Co}_{3} / \mathrm{Cu}(001)$}

In thin films the magnetic anisotropy energy can be several orders larger in magnitude than in bulk materials. The magnetostatic dipole-dipole energy also has a contribution to the magnetic anisotropy, which for thin ferromagnetic films always prefers an in-plane magnetization. In most of the theoretical studies on the thermodynamic properties of layered systems the magnetic anisotropy due to the spin-orbit coupling is described by a single-ion anisotropy [13-16]. The competition between the above two kinds of magnetic anisotropies is supposed to be responsible for different types of reorientation phase-transitions. However, the spin-orbit coupling has a non-negligible influence on the exchange couplings which is rarely included in the simulations. As demonstrated in $[17,18]$, the anisotropy of the exchange; i.e. the symmetric anisotropic part of the exchange matrix, $\mathcal{J}_{i j}^{S}$, explains the deviation of the exponent in the power-law dependence of the MAE vs. magnetization curves with respect to the theoretical predictions of Zener [19] and Callen and Callen [20] based on the symmetry of the singleion anisotropy term.

For a uniaxial system the on-site anisotropy term in Equation (1) reads as

$$
K\left(\boldsymbol{\sigma}_{i}\right)=K_{i}\left(\sigma_{i}^{z}\right)^{2}
$$

where $K_{i}$ is the the uniaxial anisotropy constant. Using a mean-field approximation the free energy of a layered system; i.e. a system underlying a two-dimensional translational invariance, can be written as [21]

$$
F=-\frac{1}{2} \sum_{p q} \mathbf{m}_{p} \mathcal{J}_{p q} \mathbf{m}_{q}-k_{B} T \ln \mathcal{Z}
$$


where the indices $p$ and $q$ label layers, $\mathbf{m}_{p}=\left\langle\sigma_{p}\right\rangle$, and $\mathcal{J}_{p q}=\sum_{j} \mathcal{J}_{p 0, q j}$. The partition function, $\mathcal{Z}$, can be expressed as follows,

$$
\begin{gathered}
\mathcal{Z}=\prod_{p} \mathcal{Z}_{p}, \\
\mathcal{Z}_{p}=2 \pi \int_{0}^{\pi} e^{-\beta\left(b_{p}^{z} \cos \vartheta+K_{p} \cos ^{2} \vartheta\right)} J_{0}\left(i \beta b_{p}^{x} \sin \vartheta\right) \sin \vartheta \mathrm{d} \vartheta,
\end{gathered}
$$

with $\beta=1 / k_{\mathrm{B}} T, b_{p}^{x(z)}=\sum_{q} J_{p q}^{x x(z z)} m_{q}^{x(z)}$ and $J_{0}$ denoting the Bessel function of zero order. Here we made use of the fact that the exchange matrix, $\mathcal{J}_{p q}$, is diagonal for an $\operatorname{fcc}(001)$ surface. It should be noted that the free energy has extrema for an in-plane and a normalto-plane ferromagnetic state [21]. The magnetizations, $\operatorname{mx}(z)_{p}$, can be found by numerically solving the following equations,

$$
\begin{aligned}
& m_{p}^{x}=\frac{2 i \pi}{\mathcal{Z}_{p}} \int_{0}^{\pi} \sin \vartheta e^{-\beta\left(b_{p}^{z} \cos \vartheta+K_{p} \cos ^{2} \vartheta\right)} J_{1}\left(i \beta b_{p}^{x} \sin \vartheta\right) \sin \vartheta \mathrm{d} \vartheta \\
& m_{p}^{z}=\frac{2 \pi}{\mathcal{Z}_{p}} \int_{0}^{\pi} \cos \vartheta e^{-\beta\left(b_{p}^{z} \cos \vartheta+K_{p} \cos ^{2} \vartheta\right)} J_{0}\left(i \beta b_{p}^{x} \sin \vartheta\right) \sin \vartheta \mathrm{d} \vartheta
\end{aligned}
$$

where $J_{1}$ is the Bessel function of the first order. Concomitantly, the free energy with respect to both directions can be determined from Equation (17) and the temperature dependent MAE is defined as,

$$
K(T)=F_{z}(T)-F_{x}(T) .
$$

As an example, for $\mathrm{Co}_{3} / \mathrm{Cu}(001)$ we determined the parameters, $J_{p q}^{x x(z z)}$ and $K_{p}$ $(p, q=1,2,3)$ in terms of the method described in Section 2. The results are summarized in Table 1. Reassuringly, the large negative isotropic exchange couplings are consistent with

Table 1. On-site uniaxial anisotropy constants and the exchange tensor for $\mathrm{Co}_{3} / \mathrm{Cu}(001) . J_{p q}^{0}$ is the isotropic exchange interaction between layer $p$ and layer $q, \Delta J_{p q}^{x x}$ and $\Delta J_{p q}^{z z}$ are the $x x$ and $z z$ element of the symmetric anisotropic exchange matrix, $\mathcal{J}_{p q}-J_{p q}^{0} \mathcal{I}$. Labels 1,2 and 3 denote the interface, central and the surface atomic Co layers, respectively.

\begin{tabular}{lrrrr}
\hline & & \multicolumn{1}{c}{1} & \multicolumn{1}{c}{2} & \multicolumn{1}{c}{3} \\
\hline$K_{p}(\mu \mathrm{eV})$ & & -91.25 & 21.29 & 269.92 \\
$J_{p q}^{0}$ & 1 & -133.74 & -99.19 & 10.85 \\
$(\mathrm{meV})$ & 2 & -99.19 & -52.42 & -114.66 \\
& 3 & 10.85 & -114.66 & -178.36 \\
$\Delta J_{p q}^{x x}$ & 1 & 29.68 & 6.60 & -70.72 \\
$(\mu \mathrm{eV})$ & 2 & 6.60 & 19.44 & -19.27 \\
& 3 & -70.72 & -19.27 & 106.35 \\
$\Delta J_{p q}^{z z}$ & 1 & -59.36 & -13.19 & 141.43 \\
$(\mu \mathrm{eV})$ & 2 & -13.19 & -38.88 & 38.54 \\
& 3 & 141.43 & 38.54 & -212.70 \\
\hline & & & &
\end{tabular}




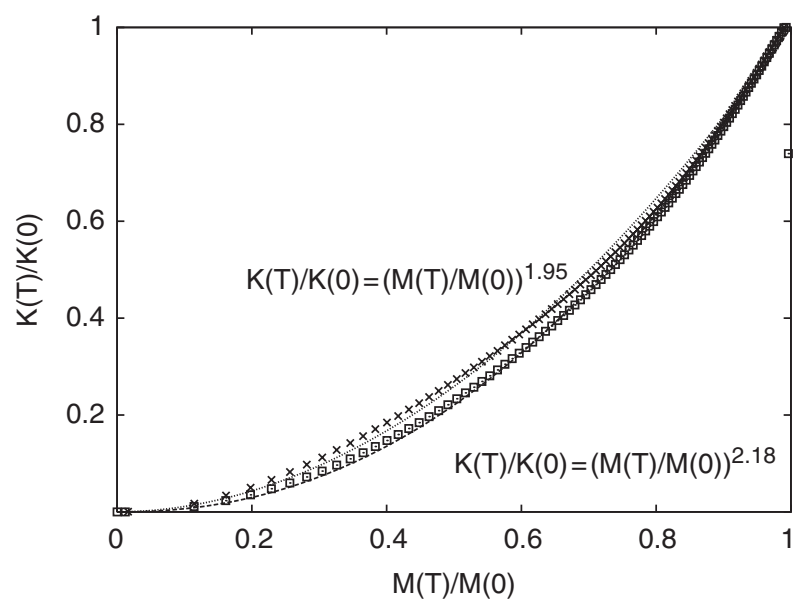

Figure 1. Normalized MAE of $\mathrm{Co}_{3} / \mathrm{Cu}(001)$ as a function of the normalized magnetization as calculated within a mean field approach. Squares denote results from a model containing both on-site anisotropies and anisotropic exchange interactions, while crosses stand for the case when the anisotropic exchange interactions are switched off. In both cases a power function shown by the solid lines has been fitted to the results.

the ferromagnetic ground state of the film. Both the on-site anisotropy and the exchange anisotropy terms prefer an in-plane magnetization. Although the on-site anisotropy constant at the interface Co layer is negative, the positive $K_{p}$ contributions at the central and, in particular, at the surface layers drive the system to an in-plane orientation at zero temperature. This tendency is even slightly enhanced due to the anisotropic exchange terms, though from Table 1 an almost perfect cancellation of contributions with different signs can be inferred. In total, we obtained a value of $K(0) \simeq 220 \mu \mathrm{eV}$.

As described in this section, within a mean field approximation we calculated the magnetization, $M(T)$, and the MAE, $K(T)$, of the Co film as a function of the temperature. In Figure 1 the normalized MAE, $K(T) / K(0)$, is plotted with respect to the normalized magnetization, $M(T) / M(0)$. Here we show also the case when the anisotropic exchange is switched off. As also shown in this figure, a power function, $K(T) \propto(M(T) / M(0))^{\alpha}$, fits well to the calculated results with $\alpha=2.18$ and 1.95 with and without taking exchange anisotropy into account, respectively. As the MAE is mainly governed by on-site anisotropy, it is not surprising that the curves are fairly similar. Remarkably, the fitted exponent is close to that predicted by the single-ion anisotropy model $[19,20]$ for $M(T) / M(0) \ll 1(\alpha=2)$, but it is different from the value of $\alpha=3$ predicted for $M(T) / M(0) \sim 1$. Our results, therefore, imply that in case of an inhomogeneous system the original predictions of the single-ion anisotropy model should be revisited.

\subsection{Effect of lattice relaxation on the magnetic pattern formation in thin films}

It has been shown recently that antisymmetric exchange interactions first predicted by Dzyaloshinsky [8,9] and Moriya [10] can be enhanced near magnetic surfaces and in nanostructures due to reduced symmetry and large strains [22,23]. The directions of Dzyaloshinsky-Moriya (DM) vectors have been investigated for several surfaces and the 
(a)

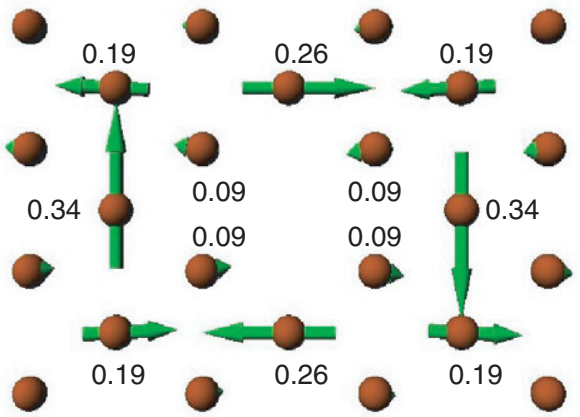

(b)

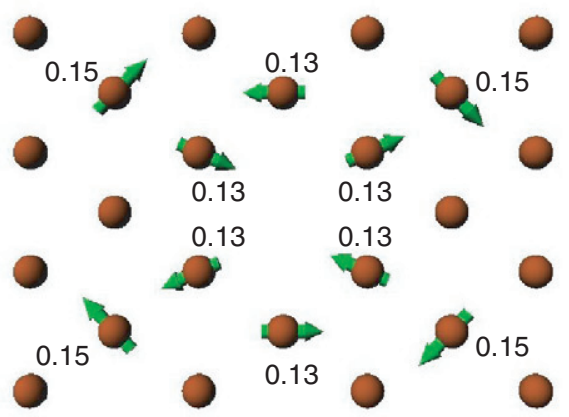

Figure 2. Sketch of the calculated $\mathrm{DM}$ vectors for $\mathrm{Fe}_{2} \mathrm{~W}(110)$ for two lattice relaxations: $26 \%$ (a) and $10 \%$ (b); see text. The values indicate the magnitudes of the DM vectors in units of $\mathrm{mRyd}$.

existence of non-collinear magnetic structures in thin films has been postulated. In terms of spin-polarized scanning tunnelling microscopy it was possible to detect a modulated magnetic structure of a Mn monolayer on a W(110) surface [24]. First principles calculations proved that such a spin-spiral like pattern formation arises from the DM interactions and even the period of the spin-spiral could be reproduced [24,25].

Nowadays, it is known that the magnetic anisotropy is quite sensitive to the geometrical relaxation of the surface. For the case of an $\mathrm{Fe}_{2} / \mathrm{W}(110)$ film, here we demonstrate that the same applies for the DM interactions and, correspondingly, to the magnetic ground state configuration of the system. There is a considerable mismatch in the lattice constants of bcc Fe and W: $a_{\mathrm{Fe}}=5.43 \mathrm{a}$.u. and $a_{\mathrm{W}}=5.96 \mathrm{a}$.u. By keeping the in-plane lattice constant of $\mathrm{W}$ fixed, we considered two cases of normal-to-plane lattice relaxations for the Fe layers, namely, a shrinking of the interlayer distances, $d_{\mathrm{W}-\mathrm{Fe}}$ and $d_{\mathrm{Fe}-\mathrm{Fe}}$, by $26 \%$ and $10 \%$ with respect to the bulk W lattice constant. The calculated DM vectors for the two systems are displayed in Figures $2 \mathrm{a}$ and $2 \mathrm{~b}$. Clearly from this figure, as the surface relaxation is decreased from $26 \%$ to $10 \%$ the magnitudes of the dominating DM interactions become about three times smaller. Remarkably also, the directions of the DM vectors differ qualitatively for the two cases.

The corresponding low temperature magnetic states obtained from Monte Carlo simulations are shown in Figure 3. Clearly, at a large lattice relaxation (26\%) the DM interactions are large enough to induce a spin-spiral modulation of the underlying ferromagnetic structure, closely resembling the modulation of the antiferromagnetic state of $\mathrm{Mn} / \mathrm{W}(110)$ [25]. Decreasing the lattice relaxation to $10 \%$, thus, decreasing also the DM interactions, a spin-spiral state with a larger period can still be recognized. We also performed calculations for smaller layer relaxations and established that at (and below) $8 \%$ relaxation the DM interactions become too weak to induce the formation of spin spirals and the normal-to-plane ferromagnetic ground state of the Fe bilayer is stabilized.

\subsection{Cr trimer on $\mathrm{Au}(111)$}

The half-filled valence configuration of $\mathrm{Cr}$ yields a large magnetic moment and strong interatomic bonding, leading in turn to magnetic frustration and complex spin phenomena. The simplest system exhibiting such properties is a trimer. 

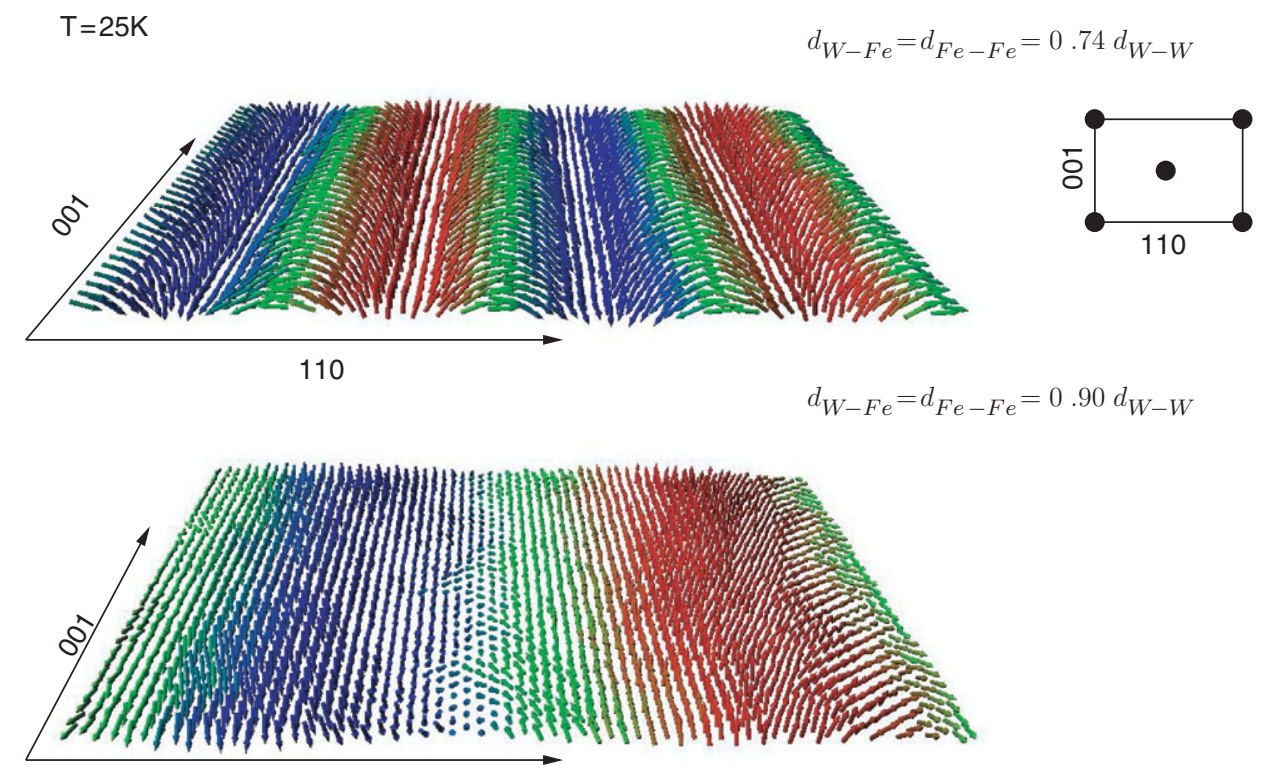

110

Figure 3. Magnetic ground state for $\mathrm{Fe}_{2} \mathrm{~W}(110)$ resulted by Monte Carlo simulations for $26 \%$ relaxation (upper figure) and 10\% relaxation (lower figure). Since the two Fe layers have almost identical configurations only the top layer is shown by the figure.

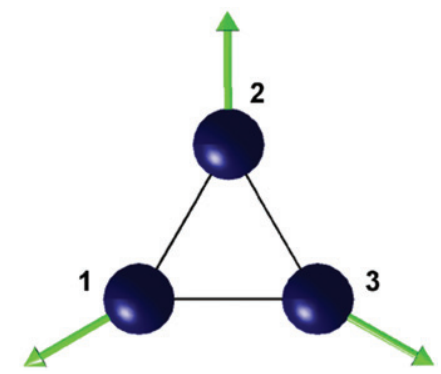

Figure 4. Magnetic ground state of the Cr cluster.

In the present study the system of three $\mathrm{Cr}$ atoms forming an equilateral triangle on $\mathrm{Au}$ (111) surface has been investigated. The magnetic properties of the cluster is described by the Heisenberg model given by Equation (1). The on-site anisotropy of the model has been chosen to be a simple quadratic form of Equation (15).

In order to calculate the electronic structure of the $\mathrm{Cr}_{3}$ clusters we applied the Embedded Cluster Green's function technique based on the Korringa-Kohn-Rostoker method (KKR-EC) [26]. The self-consistent potential has been determined for the Néel-type ground state shown in Figure 4.

Here we used two different strategies to obtain the parameters of the spin-model. On the one hand, the parameters of the model have been fitted in terms of the least-squares 
Table 2. Exchange and anisotropy matrices calculated by the torque method on the left and by fitting of the band energy on the right. All values are given in meV.

\begin{tabular}{cccccccc}
\hline & 157.94 & 2.21 & 1.12 & & 143.81 & 0.84 & -0.76 \\
$\mathcal{J}_{12}$ & -2.01 & 157.86 & 2.57 & $\mathcal{J}_{12}$ & -0.77 & 143.88 & -1.26 \\
& -1.66 & -2.26 & 157.40 & & 0.71 & 1.28 & 142.62 \\
& -0.25 & 0 & 0 & & -0.78 & 0 & 0 \\
$\mathcal{K}_{2}$ & 0 & -0.23 & 0.04 & $\mathcal{K}_{2}$ & 0 & -0.79 & -0.04 \\
& 0 & 0.04 & 0 & & 0 & -0.04 & 0 \\
\hline
\end{tabular}

(a) $\quad \mathrm{D}=3.50 \mathrm{meV}$

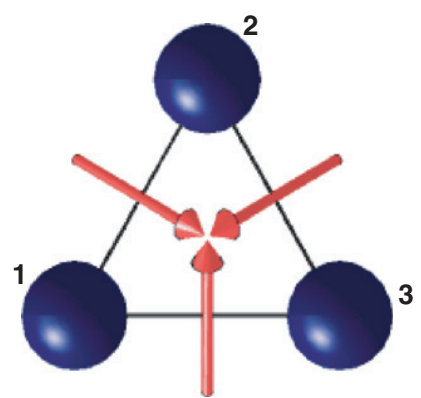

(b) $\quad \mathrm{D}=1.78 \mathrm{meV}$

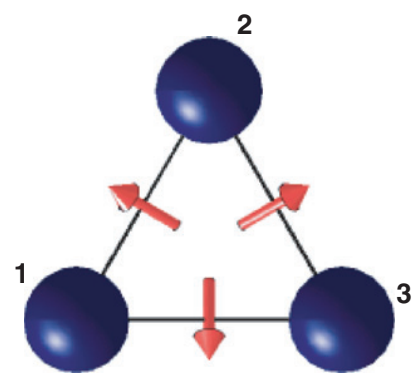

Figure 5. DM vectors obtained from the torque method (a) and from the least-square fit (b).

method to the band energies calculated for more than 2000 random magnetic configurations. The details of the procedure are given in [27]. On the other hand, the parameters have been determined by the torque method as described in Section 2. In order to find the on-site anisotropy matrices the number of parameters should be reduced using the symmetry of the system. Since the cluster has $c_{3 v}$ symmetry the coupling matrices $\mathcal{J}_{23}$ and $\mathcal{J}_{31}$ as well as the anisotropy matrices $\mathcal{K}_{1}$ and $\mathcal{K}_{3}$ can be obtained from $\mathcal{J}_{12}$ and $\mathcal{K}_{2}$ in terms of a rotation by $120^{\circ}$. Due to the mirror symmetry of the system the anisotropy matrix related to site 2 has only three non-zero elements, namely, $\mathcal{K}_{2}^{x x}, \mathcal{K}_{2}^{z z}$ and $\mathcal{K}_{2}^{y z}$. Comparing the derivatives of Equation (1) to the derivatives of the band energy, the elements of $\mathcal{K}_{2}$ can be given as

$$
\begin{aligned}
& \mathcal{K}_{2}^{x x}=\frac{1}{2}\left(\frac{\partial^{2} \mathcal{F}}{\partial \varphi_{2} \partial \varphi_{2}}-\frac{\partial^{2} \mathcal{F}}{\partial \vartheta_{2} \partial \vartheta_{2}}\right) \text { magnetization parallel to } y \text { axis } \\
& \mathcal{K}_{2}^{y y}=\frac{1}{2}\left(\frac{\partial^{2} \mathcal{F}}{\partial \varphi_{2} \partial \varphi_{2}}-\frac{\partial^{2} \mathcal{F}}{\partial \vartheta_{2} \partial \vartheta_{2}}\right) \quad \text { magnetization parallel to } x \text { axis } \\
& \mathcal{K}_{2}^{y z}=\frac{1}{2} \frac{\partial^{2} \mathcal{F}}{\partial \varphi_{2} \partial \vartheta_{2}} \quad \text { magnetization parallel to } x \text { axis }
\end{aligned}
$$

The corresponding results are summarized in Table 2. Both methods predict strong antiferromagnetic couplings between the $\mathrm{Cr}$ atoms. The difference between the isotropic 
exchange interactions provided by the two methods is less than $10 \%$. The anisotropic exchange interactions are much more different. Since the least-square fit procedure is regarded as a more exact mapping of the first-principles band energy, the torque method overestimates the on-site anisotropy and the anisotropic exchange by about a factor of two. Even the directions of the DM vectors shown in Figure 5 are different, however, their components perpendicular to the plane, responsible for the chirality of the magnetic ground state $[27,28]$, have the same directions. Reassuringly, therefore, the two sets of parameters resulted in the same magnetic ground state configuration.

\section{Acknowledgements}

Financial support was provided by the Hungarian National Scientific Research Foundation (contract no. OTKA T068312, F68726 and NF061726).

\section{References}

[1] L.M. Sandratskii, J. Phys. Condensed Matter 3 (1991) p.8565.

[2] S.V. Halilov, H. Eschrig, A.Y. Perlov et al., Phys. Rev. B 58 (1998) p.293.

[3] O. Grotheer, C. Ederer and M. Fähnle, Phys. Rev. B 63 (2002) p.1000401.

[4] Ph. Kurz, G. Bihlmayer, K. Hirai et al., Phys. Rev. Lett. 86 (2001) p.1106.

[5] A.I. Liechtenstein, M.I. Katsnelson, V.P. Antropov et al., J. Magn. Magn. Mater. 67 (1987) p.65.

[6] A. Oswald, R. Zeller, P.J. Braspenning et al., J. Phys. F: Met. Phys. 15 (1985) p.193.

[7] L. Udvardi, L. Szunyogh, K. Palotás et al., Phys. Rev. B 68 (2003) p.104436.

[8] I. Dzyaloshinsky, Sov. Phys. JETP 5 (1957) p.1259.

[9] I. Dzyaloshinsky, J. Phys. Chem. Solids 4 (1958) p.241.

[10] T. Moriya, Phys. Rev. 120 (1960) p.91.

[11] L. Szunyogh, B. Újfalussy and P. Weinberger, Phys. Rev. B 51 (1995) p.9552.

[12] P. Lloyd, Proc. Phys. Soc. London 90 (1967) p.207.

[13] A. Hucht and K.D. Usadel, J. Magn. Magn. Mater. 156 (1996) p.423.

[14] A. Hucht and K.D. Usadel, Phys. Rev. B 66 (2002) p.024419.

[15] A. Hucht and K.D. Usadel, Phys. Rev. B 55 (1997) p.12309.

[16] Liu Yinga, Chen Nanxiana, Zhao Hongminb et al., Sol. Stat. Comm. 126 (2003) p.223.

[17] J.B. Staunton, L. Szunyogh, Á. Buruzs et al., Phys. Rev. B 74 (2006) p.144411.

[18] Á. Buruzs, P. Weinberger, L. Szunyogh et al., Phys. Rev. B 76 (2007) p.064417.

[19] C. Zener, Phys. Rev. 96 (1954) p.1335.

[20] H.B. Callen and E. Callen, J. Phys. Chem. Solids 27 (1966) p.1271.

[21] L. Udvardi, L. Szunyogh, A. Vernes et al., Phil. Mag. B 81 (2001) p.613.

[22] A. Fert, in Materials Science Forum, Vol. 59-60, A. Chamberod and J. Hillairat eds., Transtech Publications, Aedermannsdorf, 1990, p.439.

[23] A. Crépieux and C. Lacroix, J. Magn. Magn. Mater. 182 (1998) p.341.

[24] M. Bode, M. Heide, K. Bergmann et al., Nature 447 (2007) p.193.

[25] L. Udvardi, A. Antal, L. Szunyogh et al., Physica B: Condensed Matter 403 (2008) p.402.

[26] B. Lazarovits, L. Szunyogh and P. Weinberger, Phys. Rev. B 65 (2002) p.104441.

[27] A. Antal, L. Udvardi, B. Lazarovits et al., Phys. Rev. B 77 (2008) p.174429.

[28] A. Antal, L. Udvardi and B. Újfalussy, J. Magn. Magn. Mater. 316 (2007) p.118. 